\title{
Allergic contact dermatitis to dorzolamide and benzalkonium chloride
}

\author{
Diego Orsini ${ }^{1}$, Andrea D’Arino², Flavia Pigliacelli ${ }^{2}$, Chiara Assorgi ${ }^{2}$, Alessandra Latini ${ }^{1}$, Antonio Cristaudo ${ }^{1}$
}

${ }^{1}$ IFO, Dermatological Institute S. Gallicano, IRCCS, Rome, Italy

2Faculty of Medicine, University of Rome "Sapienza", Rome, Italy
Adv Dermatol Allergol 2018; XXXV (5): 538-539

DOI: https://doi.org/10.5114/ada.2018.73859
Dorzolamide is a topical carbonic anhydrase II and XII inhibitor that can be administered topically, directly into the eye, as a $2 \%$ water solution [1]. Common side effects are conjunctivitis, erythema and eyelid edema [2]. To the best of our knowledge, true contact dermatitis to Dorzolamide is rare and only a few cases have been reported in the literature [3]. We hereby report a case of contact dermatitis to both Dorzolamide and its excipient benzalkonium chloride.

A 64-year-old man presented to our Dermatology Department due to the presence of bilateral palpebral erythema and swelling for 2 months. Upon clinical examination, both the superior and inferior eyelids appeared erythematous with fine scaling (Figure 1). The reaction was symmetrical and extended to the zygomatic arch on either side with no visible involvement of the conjunctiva. The only symptom the patient complained of was intense itching.

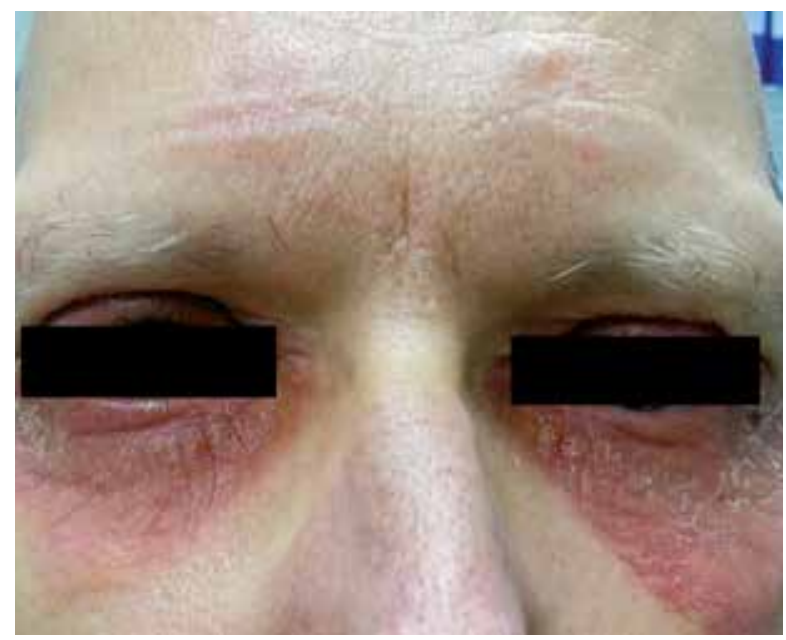

Figure 1. Both the superior and inferior eyelids appeared erythematous with fine scaling
Past dermatologic history was negative for atopic dermatitis, psoriasis or any other dermatologic condition. The patient's medical history included type 2 diabetes mellitus, in treatment with metformin $500 \mathrm{mg}$ tid, and a new diagnosis of open-angle glaucoma only recently in treatment with dorzolamide $2 \%$ eye drops ( 1 drop tid). All the previous therapies including low-power topical corticosteroids and topical pimecrolimus had given only mild results with constant relapse of the symptoms.

Given the suspicion of contact dermatitis, patch tests and patch by patch with $2 \%$ Dorzolamide eye drops were carried out according to the SIDAPA guidelines. The tests were removed after $48 \mathrm{~h}$ and standard readings were carried out at 48 (T1) and $72 \mathrm{~h}$ (T2). The patch by patch with dorzolamide $2 \%$ eye drops in paraffin was positive, with erythema and edema (++) at T1 and vesico-pustules (+++) at T2, while the standard patch test was positive to benzalkonium chloride 0.1\% (+ at T1 and ++ at T2) (Figure 2).

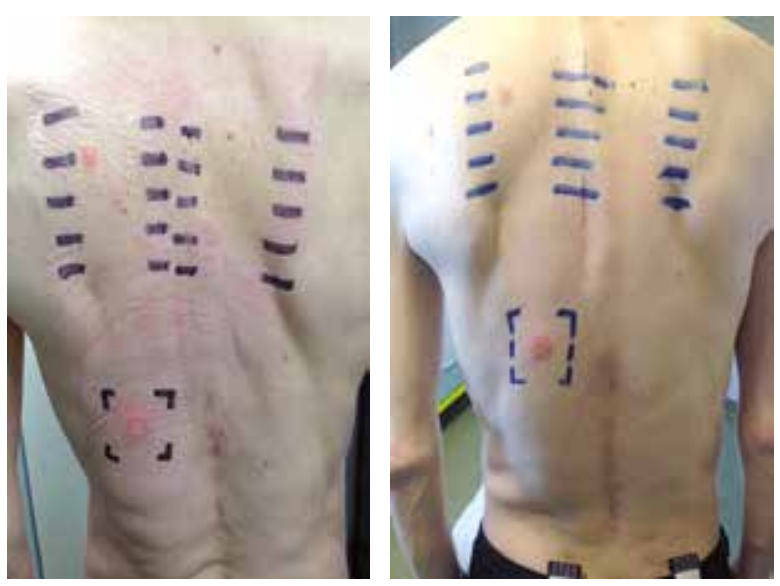

Figure 2. The patch by patch with dorzolamide $2 \%$ eye drops in paraffin was positive, with erythema and edema (++) at T1 and vesico-pustules (+++) at T2, while the standard patch test was positive to benzalkonium chloride $0.1 \%$ (+ at T1 and ++ at T2)

Address for correspondence: Diego Orsini MD, UOSD Dermatologia MST, Ambientale, Tropicale e Immigrazione, Istituto Dermatologico San Gallicano (IRCCS), Via Elio Chianesi 53, Rome, Italy, e-mail: diegorsini@gmail.com Received: 4.10.2017, accepted: 3.11.2017. 
Considering the test results we diagnosed the patient with allergic contact dermatitis due to both the active drug, Dorzolamide, and the excipient benzalkonium chloride $0.1 \%$. The eye drops were suspended and switched to an alternative therapy as per the ophthalmologist's advice and complete remission of symptoms was obtained in 2 weeks. Topically applied carbonic anhydrase inhibitors, such as Dorzolamide, in eye drop solutions are commonly used to treat glaucoma [4]. A few cases of true allergic contact dermatitis to Dorzolamide have been reported $[5,6]$ but, to our knowledge, this is the first report of an allergy to both the active drug and the excipient. Moreover, contact sensitization to benzalkonium chloride alone is rare [7].

We believe our case is interesting because benzalkonium chloride is the most common preservative in glaucoma medications [8] and the combination of sensitization to both Dorzolamide and its excipient has rarely been reported in the literature. For this reason, it should be considered when evaluating any form of suspect contact dermatitis in a patient in treatment with any kind of topical therapy for glaucoma as in our case. Recognition of the offending agent is of fundamental importance to obtain complete clinical resolution of the disease.

\section{Conflict of interest}

The authors declare no conflict of interest.

\section{References}

1. Scozzafava A, Supuran CT. Glaucoma and the applications of carbonic anhydrase inhibitors. Subcell Biochem 2014; 75 : 349-59.

2. Pfeiffer N. Dorzolamide: development and clinical application of a topical carbonic anhydrase inhibitor. Surv Ophthalmol 1997; 42: 137-51.

3. Lee SJ, Kim M. Allergic contact dermatitis caused by dorzolamide eyedrops. Clin Ophthalmol 2015; 9: 575-7.

4. Loftsson T, Jansook P, Stefansson E. Topical drug delivery to the eye: dorzolamide. Acta Ophthalmol 2012; 90: 603-8.

5. Shimada M, Higaki Y, Kawashima M. Allergic contact dermatitis due to dorzolamide eyedrops. Contact Dermatitis 2001; 45: 52.

6. Linares Mata T, Pardo Sanchez J, de la Cuadra Oyanguren J. Contact dermatitis caused by allergy to dorzolamide. Contact Dermatitis 2005; 52: 111-2.

7. Dao H Jr, Fricker C, Nedorost ST. Sensitization prevalence for benzalkonium chloride and benzethonium chloride. Dermatitis 2012; 23: 162-6.

8. Rasmussen CA, Kaufman PL, Kiland JA. Benzalkonium chloride and glaucoma. J Ocul Pharmacol Ther 2014; 30: 163-9. 